\title{
HYPERFINITE EXTENSIONS OF BOUNDED OPERATORS ON A SEPARABLE HILBERT SPACE $\left({ }^{1}\right)$
}

BY

\author{
L. C. MOORE, JR.
}

ABSTRACT. Let $H$ be a separable Hilbert space and $\hat{H}$ the nonstandard hull of $H$ with respect to an $\aleph_{1}$-saturated enlargement. Let $S$ be a*finite dimensional subspace of ${ }^{*} H$ such that the corresponding hyperfinite dimensional subspace $\hat{S}$ of $\hat{H}$ contains $H$. If $T$ is a bounded operator on $H$, then an extension $\hat{A}$ of $\boldsymbol{T}$ to $\hat{S}$ where $\hat{A}$ is obtained from an internal *-linear operator on $S$ is called a hyperfinite extension of $T$. It is shown that $T$ has a compact (selfadjoint) hyperfinite extension if and only if $T$ is compact (selfadjoint). However $T$ has a normal hyperfinite extension if and only if $T$ is subnormal. The spectrum of a hyperfinite extension $\hat{A}$ equals the point spectrum of $\hat{A}$, and if $T$ is quasitriangular, $A$ can be chosen so that the spectrum of $\hat{A}$ equals the spectrum of $T$. A simple proof of the spectral theorem for bounded selfadjoint operators is given using a hyperfinite extension.

1. Preliminaries. The nonstandard hull of a normed space was introduced by Luxemburg in [9]. The properties of these spaces have been investigated by Henson, Cozart, and the author in [6], [7], [2], and [8]. We review the relevant definitions here.

Let $(E,\|\cdots\|)$ be a normed space and let $* M$ be an $\aleph_{1}$-saturated enlargement [9] of a set theoretical structure $M$ which contains $(E,\|\cdots\|)$. An element $p \in{ }^{*} E$ is called finite if $*\|p\|$ is a finite element of ${ }^{*} R$ and the set of finite elements of ${ }^{*} E$ is denoted by $\operatorname{fin}\left({ }^{*} E\right)$. The monad of 0 is defined by

$$
\mu(0)=\left\{p: p \in{ }^{*} E \text { and } *\|p\| \text { is infinitesimal }\right\} .
$$

Both fin $\left({ }^{*} E\right)$ and $\mu(0)$ are vector spaces over the same field as $E$. We denote the quotient vector space fin $\left({ }^{*} E\right) / \mu(0)$ by $\hat{E}$ and the canonical quotient mapping of fin $\left({ }^{*} E\right)$ onto $\hat{E}$ by $\pi$. A norm may be defined on $\hat{E}$ by letting $\|\pi(p)\|$ be st $*\|p\|$, where st is the standard part operator on ${ }^{*} R$. The normed space $(\hat{E}$,

Received by the editors February 24, 1975.

AMS (MOS) subject classifications (1970). Primary 02H25, 47Axx; Secondary 47B20.

Key words and phrases. Nonstandard analysis, Hilbert space, operator, spectrum, subnormal.

(1) The results in this paper were presented at the 1974 Oberwolfach Nonstandard Analysis Meeting which was dedicated to the memory of Abraham Robinson. 
$\|\cdots\|)$ is called the nonstandard hull of $(E,\|\cdots\|)$ (with respect to *M).

It is shown in [9] that under the assumption of $\aleph_{1}$-saturation, $(\hat{E},\|\cdots\|)$ is a Banach space. Further $(E,\|\cdots\|)$ may be considered as a subspace of $(\hat{E}$, $\|\cdots\|)$ by identifying $x \in E$ with $\left.\pi^{*} x\right)$. In the case that the original space is a Hilbert space $H$ with inner product $\langle\ldots, \ldots\rangle$, the nonstandard hull $\hat{H}$ is also a Hilbert space with the extended inner product $\langle\pi(p), \pi(q)\rangle=\operatorname{st}(*\langle p, q\rangle)$.

If $S$ is a $*$-finite dimensional subspace of $* E$, then $\hat{S}=\{\pi(p): p \in S \cap$ fin $\left.\left({ }^{*} E\right)\right\}$ is called a hyperfinite dimensional subspace of $E$ [7]. We are interested in hyperfinite dimensional subspaces $\hat{S}$ such that $\hat{S} \supseteq E$. It is easy to show that since $* M$ is an enlargement, such hyperfinite dimensional subspaces exist. If $H$ is a separable Hilbert space and $\left\{e_{1}, e_{2}, \ldots\right\}$ is an orthonormal basis, let $S=$ ${ }^{*}$-span $\left\{{ }^{*} e_{1},{ }^{*} e_{2}, \ldots,{ }^{*} e_{\omega}\right\}$ where $\omega$ is an infinite positive integer. It is easy to verify that $\hat{S}$ is a hyperfinite dimensional subspace containing $H$.

Now let $H$ be a separable Hilbert space. Let $C$ denote the field of complex numbers, $Z$ the set of integers, and $N$ the set of positive integers. Let st denote the standard part operator on ${ }^{*} C$ and if $\alpha, \beta \in{ }^{*} C$ and $\alpha-\beta$ is infinitesimal, we write $\alpha={ }_{1} \beta$. If $p, q \in{ }^{*} H$ and $*\|p-q\|={ }_{1} 0$, we write $p \sim q$. Similarly if $A$ and $D$ are *-bounded operators on $* H$, we write $A \sim D$ if $*\|A-D\|={ }_{1} 0$. Let $B$ denote the closed unit ball in $H$. Finally all projections considered on $H$ are assumed to be orthogonal, and in order to avoid confusion, the adjoint of an operator $T$ is denoted by $T^{\#}$.

Let $S$ be a *-finite dimensional subspace of ${ }^{*} H$ such that $\hat{S} \supseteq H$ and let $2 \nVdash(S)$ be the algebra of *-linear operators $A$ on $S$ such that *\|A\| is a finite element of ${ }^{*} R$. If $A \in \mathscr{Q}(S)$, it is easy to verify that $A$ induces a bounded operator $\hat{A}$ on $\hat{S}$ by setting

$$
A(\pi(p))=\pi(A(p))
$$

The following result is elementary and the proof is left to the reader.

LEMma 1.1. Let $S$ be a ${ }^{*}$-finite dimensional subspace of ${ }^{*} H$ such that $\hat{S}$ $\supseteq H$, let $A, D \in \mathfrak{A}(S)$, and $\lambda \in$ fin $\left({ }^{*} C\right)$. Then

(i) $\|\hat{A}\|=\operatorname{st}(*\|A\|)$,

(ii) $\widehat{A+D}=\hat{A}+\hat{D}$,

(iii) $\widehat{\lambda A}=\operatorname{st}(\lambda) \hat{A}$,

(iv) $\widehat{A D}=\hat{A} \hat{D}$,

(v) $(\hat{A})^{\#}=\left(\widehat{A^{\#}}\right)$,

(vi) if $A$ is $a^{*}$-orthogonal projection on $S$, then $\hat{A}$ is an orthogonal projection on $\hat{S}$.

Now let $T$ be a bounded operator on $H$.

Definition 1.2 . Let $S$ be a *-finite dimensional subspace of ${ }^{*} H$ such that 
$\hat{S} \supseteq H$ and let $A \in \mathscr{U}(S)$. If $\hat{A}$ restricted to $H$ is $T$, then $\hat{A}$ is called a hyperfinite extension of $T$.

Let $S$ be a ${ }^{*}$-finite dimensional subspace of ${ }^{*} H$ such that $\hat{S} \supseteq H$ and let $P_{S}$ be the *-orthogonal projection of ${ }^{*} H$ onto $S$. Denote the restriction of $P_{S}^{*} T$ to $S$ by ${ }^{*} T_{S}$. Since $*\left\|P_{S}\right\|=1$, we have ${ }^{*}\left\|T_{S}\right\| \leqslant *\left\|P_{S} * T\right\| \leqslant *\|* T\|=$ $\|T\|$, so $* T_{S} \in \mathscr{Q}(S)$. We denote the operator $\widehat{ }^{*} \widehat{T}_{S}$ on $\hat{S}$ by $\hat{T}_{S}$.

LEMMA 1.3. If $S$ is a hyperfinite dimensional subspace of $\hat{H}$ such that $\hat{S}$ $\supseteq H$, then $\hat{T}_{S}$ is a hyperfinite extension of $T$ and $\left\|\hat{T}_{S}\right\|=\|T\|$.

Proof. Let $x \in H$. Then by assumption there exist $p, q \in S$ such that $p \sim{ }^{*} x$ and $q \sim{ }^{*}(T x)$. Then ${ }^{*} T p \sim{ }^{*} T\left({ }^{*} x\right)={ }^{*}(T x) \sim q$ and ${ }^{*} P_{S} * T(p) \sim{ }^{*} P_{S} q$ $=q$. Thus $\hat{T}_{S}(x)=\hat{T}_{S}(\pi(p))=\pi\left({ }^{*} T_{S} p\right)=\pi(q)=T x$ and $\hat{T}_{S}$ extends $T$. By the comments above $\left\|\hat{T}_{S}\right\| \leqslant\|T\|$ and since $\hat{T}_{S}$ extends $T$ we have $\|T\| \leqslant\left\|\hat{T}_{S}\right\|$. Thus $\|T\|=\left\|\hat{T}_{S}\right\|$.

DEFINITION 1.4. Let $S$ be a hyperfinite dimensional subspace of $H$ such that $\hat{S} \supseteq H$. Then $\hat{T}_{S}$ is called the standard hyperfinite extension of $T$ with respect to $S$.

2. Selfadjoint, compact, and normal operators.

Lemma 2.1. $\left(\hat{T}_{S}\right)^{\#}=\widehat{\left(T^{\#}\right)_{S}}$ for any bounded operator $T$ and any *-finite dimensional subspace $S$ of ${ }^{*} H$ such that $\hat{S} \supseteq H$.

Proof. The operator ${ }^{*} T_{S}$ is the restriction of $P_{S}{ }^{*} T P_{S}$ to $S$ and $\left({ }^{*} T_{S}\right)^{\#}$ is the restriction of $P_{S}\left({ }^{*} T\right)^{\#} P_{S}=P_{S}{ }^{*}\left(T^{\#}\right) P_{S}$ to $S$. Thus $\left({ }^{*} T_{S}\right)^{\#}={ }^{*}\left(T^{\#}\right)_{S}$ and the result follows from Lemma 1.1(v).

LEMMA 2.2. The following conditions are equivalent:

(i) $T$ is selfadjoint,

(ii) some hyperfinite extension of $T$ is selfadjoint,

(iii) every standard hyperfinite extension of $T$ is selfadjoint.

Proof. Clearly (iii) implies (ii) and since the restriction of a selfadjoint operator to an invariant subspace is again selfadjoint, (ii) implies (i). Finally if $T$ is selfadjoint, then Lemma 2.1 implies that every standard hyperfinite extension is again selfadjoint, i.e., (i) implies (iii).

LEMMA 2.3. The following conditions are equivalent:

(i) $T$ is compact,

(ii) some hyperfinite extension of $T$ is compact,

(iii) every standard hyperfinite extension of $T$ is compact.

Proof. Clearly (iii) implies (ii) and since the restriction of a compact operator to an invariant subspace is again compact, (ii) implies (i). To show 
that (i) implies (iii) assume $T$ is compact and $\hat{S}$ is a hyperfinite dimensional subspace of $\hat{H}$ containing $H$. Let $\epsilon>0$. Then there exists a finite set $\left\{x_{1}, x_{2}\right.$, $\left.\ldots, x_{n}\right\}$ in $H$ such that $T(B) \subseteq \bigcup_{i=1}^{n}\left\{x \in H:\left\|x-x_{i}\right\|<\epsilon\right\}$. Hence ${ }^{*} T\left(^{*} B\right) \subseteq$ $\bigcup_{i=1}^{n}\left\{p \in{ }^{*} H:{ }^{*}\left\|p-{ }^{*} x_{i}\right\|<\epsilon\right\}$ and it follows that $\hat{T}_{S}\left(\hat{B}_{S}\right) \subseteq \bigcup_{i=1}^{n}\{x \in \hat{S}$ : $\left.\left\|x-x_{i}\right\| \leqslant \epsilon\right\}$ where $\hat{B}_{S}$ is the closed unit ball in $\hat{S}$. Thus $\hat{T}_{S}$ is compact.

Let $\omega$ be an infinite positive integer, then the (external) cardinality of the set $\{1,2, \ldots, \omega\}$ is always greater than or equal to $c$. To see this note that for each $r \in[0,1]$ there exists $k_{r} \in\{1,2, \ldots, \omega\}$ such that $\operatorname{st}\left(k_{r} / \omega\right)=r$. We use this observation in the proof of

THEOREM 2.4. Let $\hat{S}$ be a hyperfinite dimensional subspace of $\hat{H}$ containing $H$.

(i) If $A \in \mathscr{U}(S)$, then $\hat{A}$ is compact if and only if $\hat{A}(\hat{S})$ is separable.

(ii) $T$ is compact if and only if $\hat{T}_{S}(\hat{S}) \subseteq H$.

Proof. (i) Clearly if $\hat{A}$ is compact then $\hat{A}(\hat{S})$ is separable.

On the other hand if $\hat{A}$ is not compact then for some $\epsilon>0$ there exists a sequence $\left\{x_{n}\right\}$ in $\hat{S}$ such that $\left\|x_{n}\right\| \leqslant 1$ for all $n$ and $\left\|\hat{A} x_{n}-\hat{A} x_{m}\right\|>\epsilon$ for all $n \neq m$. For each $n$ pick $p_{n} \in S \cap * B$ such that $\pi\left(p_{n}\right)=x_{n}$ and using the $\aleph_{1}$ saturation extend this to an internal sequence defined on ${ }^{*} N$. Now there exists an infinite positive integer $\omega$ such that $p_{k} \in{ }^{*} B \cap S$ for all $k \leqslant \omega$ and $*\left\|A p_{k}-A p_{j}\right\|>\epsilon$ for all $1 \leqslant j<k \leqslant \omega$. Thus $\left\{\hat{A}\left(\pi\left(p_{k}\right)\right): 1 \leqslant k \leqslant \omega\right\}$ is a set in $\hat{A}(\hat{S})$ with cardinality the same as the cardinality of $\{1,2, \ldots, \omega\}$ and such that every pair of elements is at least $\epsilon$ apart. Since $\{1,2, \ldots, \omega\}$ is not countable, this implies $\hat{A}(\hat{S})$ is not separable.

(ii) If $\hat{T}_{S}(\hat{S}) \subseteq H$, then $\hat{T}_{S}$ is compact by (i) above and $T$ is compact by Lemma 2.3. On the other hand if $T$ is compact, then $p \in$ fin $\left({ }^{*} H\right)$ implies that for some $x \in H$ we have ${ }^{*} T p \sim{ }^{*} x[10]$. It follows easily that $\hat{T}_{S}(\hat{S}) \subseteq H$.

The situation for normal operators is more complicated. We recall a few definitions.

DEFinition 2.5. (i) $T$ is said to be quasidiagonal if there is an increasing sequence $\left\{E_{n}\right\}$ of projections of finite rank such that $E_{n} \rightarrow 1$ (strongly) and $\left\|T E_{n}-E_{n} T\right\| \rightarrow 0$.

(ii) $T$ is said to be quasitriangular if there is an increasing sequence $\left\{E_{n}\right\}$ of projections of finite rank such that $E_{n} \rightarrow 1$ (strongly) and $\left\|T E_{n}-E_{n} T E_{n}\right\|$ $\rightarrow 0$.

Both definitions are due to Halmos ([4] and [5]). Note that every quasidiagonal operator is quasitriangular. Recall that a closed subspace of $H$ is said to be reducing for $T$ if it is invariant for both $T$ and $T^{\#}$. Thus $T$ is quasidiagonal (quasitriangular) if and only if there are sufficiently many "almost reducing" ("almost invariant") finite dimensional subspaces. It is easy to show [5] that 
compact operators and normal operators are quasidiagonal.

The following theorem is proved by Halmos [4] in the quasitriangular case. The proof in the quasidiagonal case is analogous.

Theorem 2.6 (HAlmos). (i) $T$ is quasidiagonal if and only if there is a sequence $\left\{E_{n}\right\}$ of projections of finite rank such that $E_{n} \rightarrow 1$ (strongly) and $\left\|T E_{n}-E_{n} T\right\| \rightarrow 0$.

(ii) $T$ is quasitriangular if and only if there is a sequence $\left\{E_{n}\right\}$ of projections of finite rank such that $E_{n} \rightarrow 1$ (strongly) and $\left\|T E_{n}-E_{n} T E_{n}\right\| \rightarrow 0$.

In other words both definitions in 2.4 are unchanged if the requirement that the sequence of projections be increasing is dropped. The following lemma is a simple consequence of Theorem 2.5 .

LEMMA 2.7. (i) $T$ is quasidiagonal if and only if there exists $a *$-finite dimensional subspace $S$ of ${ }^{*} H$ such that $\hat{S} \supseteq H$ and ${ }^{*} T P_{S} \sim P_{S} * T$.

(ii) $T$ is quasitriangular if and only if there exists $a *$-finite dimensional subspace $S$ of ${ }^{*} H$ such that $\hat{S} \supseteq H$ and ${ }^{*} T P_{S} \sim P_{S}{ }^{*} T P_{S}$.

LEMMA 2.8. T has a normal standard hyperfinite extension if and only if $T$ is normal.

Proof. Assume that $\hat{T}_{S}$ is a normal standard hyperfinite extension. Since

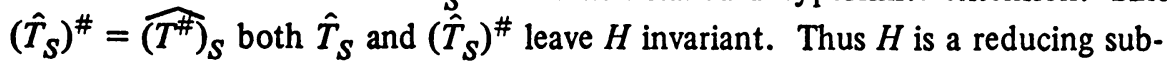
space for $\hat{T}_{S}$ and since $T$ is the restriction of $\hat{T}_{S}$ to $H, T$ is also normal.

Assume $T$ is normal. Then $T$ is quasidiagonal and by Lemma 2.7 there exists a *-finite dimensional subspace $S$ of ${ }^{*} H$ such that $\hat{S} \supseteq H$ and ${ }^{*} T P_{S} \sim P_{S}{ }^{*} T$. Now $\widehat{\left(T_{S}\right)^{\#}}=\widehat{\left(T^{\#}\right)_{S}}$ by Lemma 2.1 , so $\hat{T}_{S}\left(\hat{T}_{S}\right)^{\#}=\hat{T}_{S} \widehat{\left(T^{\#}\right)_{S}}=\left({ }^{*} T_{S}{ }^{*}\left(T^{\#}\right)_{S}\right)^{*}$. Similarly $\left(\hat{T}_{S}\right)^{\#} \hat{T}_{S}=\left({ }^{*}\left(T^{\#}\right)_{S}{ }^{*} T_{S}\right)^{\wedge}$. Thus in order to show that $\hat{T}_{S}$ is normal, it is sufficient to show that $\left(P_{S}{ }^{*} T P_{S}\right)\left(P_{S} *\left(T^{\#}\right) P_{S}\right) \sim\left(P_{S} *\left(T^{\#}\right) P_{S}\right)\left(P_{S}{ }^{*} T P_{S}\right)$. But since ${ }^{*} T P_{S} \sim P_{S}{ }^{*} T$, we have $\left(P_{S}{ }^{*} T P_{S}\right)\left(P_{S} *\left(T^{\#}\right) P_{S}\right) \sim P_{S}{ }^{*} T^{*}\left(T^{\#}\right) P_{S}=$ $P_{S} *\left(T T^{\#}\right) P_{S}=P_{S} *\left(T^{\#} T\right) P_{S} \sim\left(P_{S}^{*}\left(T^{\#}\right) P_{S}\right)\left(P_{S} * T P_{S}\right)$.

EXAMPLE 2.9. Even if $T$ is normal it does not follow that every standard hyperfinite extension of $T$ is normal. For example let $T$ be the two-sided shift, i.e., $T$ is defined on $l_{2}(Z)$ and $T e_{k}=e_{k+1}$ where $\left\{e_{k}: k \in Z\right\}$ is the usual orthonormal basis on $l_{2}(Z)$. Pick $\omega$ an infinite positive integer and let $S=*$-span of $\left\{{ }^{*} e_{k}:-\omega \leqslant k \leqslant \omega\right\}$. Then ${ }^{*} T\left({ }^{*} e_{\omega}\right)={ }^{*} e_{\omega+1}$, so ${ }^{*} T_{S}\left({ }^{*} e_{\omega}\right)=0$ and ${ }^{*}\left(T^{\#}\right)_{S}{ }^{*} T_{S}\left({ }^{*} e_{\omega}\right)=0$. But ${ }^{*} T_{S}{ }^{*}\left(T^{\#}\right)_{S}\left({ }^{*} e_{\omega}\right)={ }^{*} T_{S}\left({ }^{*} e_{\omega-1}\right)={ }^{*} e_{\omega}$. Thus $\left(\hat{T}_{S}\right)^{\#} \hat{T}_{S}\left(\pi\left(e_{\omega}\right)\right) \neq 0$ and $\hat{T}_{S}\left(\hat{T}_{S}\right)^{\#}\left(\pi\left(e_{\omega}\right)\right)=0$, hence $\hat{T}_{S}$ is not normal.

Recall that an operator $T$ is said to be subnormal if it has a normal extension. It is easy to verify that an operator on a separable Hilbert space is subnormal if and only if it has a normal extension to a separable Hilbert space. 
THEOREM 2.10. T has a normal hyperfinite extension if and only if $T$ is subnormal.

Proof. Assume $T$ is subnormal. Then we may assume $H$ is a subspace of a separable Hilbert space $K$ and that $T$ has a normal extension $Q$ on $K$. Further since $K$ is separable we may assume $K \in M$. Since $K$ is normal it is quasidiagonal, so there is a *-finite dimensional subspace $V$ of ${ }^{*} K$ such that $\hat{V} \supseteq K$ and ${ }^{*} Q P_{V} \sim$ $P_{V} * Q$.

Since both $H$ and $K$ are separable, there is an isometry $\Phi$ of $K$ onto $H$. Let $S$ be the image of $V$ under ${ }^{*} \Phi$. Then $S$ is a *-finite dimensional subspace of ${ }^{*} H$. We assert that $\hat{S} \supseteq H$. To see this let $x \in H$ and let $z=\Phi^{-1} x$. Since $\hat{V} \supseteq K$ there exists $q \in V$ such that $q \sim *^{*} z$. It follows that $\Phi q \sim \Phi^{*} z={ }^{*}(\Phi z)={ }^{*} x$, and so $\hat{S} \supseteq H$.

Next let $\left\{e_{k}: k \in N\right\}$ be a fixed orthonormal basis in $H$. For each $k \in N$, pick $p_{k} \in S$ and $q_{k} \in V$ such that $p_{k} \sim{ }^{*} e_{k}$ and $q_{k} \sim{ }^{*} e_{k}$. For $k, j \in N$ with $k \neq j$, we have $*\left\langle p_{k}, p_{j}\right\rangle={ }_{1}{ }^{*}\left\langle e_{k},{ }^{*} e_{j}\right\rangle=0$, thus by applying the Gram-Schmidt process to $\left\{p_{k}: k \in N\right\}$ we may assume $*\left\langle p_{k}, p_{j}\right\rangle=0$ for $k \neq j$. Similarly we may assume $*\left\langle q_{k}, q_{j}\right\rangle=0$ for $k, j \in N$ and $k \neq j$.

Now for each $n \in N$ let $A_{n}$ be the set of all *-linear mappings $\theta$ of $V$ onto $S$ such that $\theta q_{k}=p_{k}$ for $k=1,2, \ldots, n$ and $1-1 / n \leqslant *\|\theta\| \leqslant 1+1 / n$. Clearly each set $A_{n}$ is nonempty and internal. Hence by $\aleph_{1}$-saturation there exists $\Psi \in \bigcap_{n \in N} A_{n}$; so $\Psi$ is a *-linear mapping of $V$ onto $S$ such that $\Psi\left(q_{k}\right)=$ $\dot{p}_{k}$ for all $k \in N$ and $*\|\Psi\|==_{1} 1$. Note that if $q$ and $q^{\prime} \in V \cap$ fin $\left({ }^{*} K\right)$ then it follows from the parallelogram identity that $*\left\langle q, q^{\prime}\right\rangle={ }_{1} *\left\langle\Psi q, \Psi q^{\prime}\right\rangle$.

We define a mapping $A$ of $S$ into $S$ by $A p=\Psi P_{V}^{*} Q \Psi^{-1} p$. Clearly $A$ is *-linear and $*\|A\| \leqslant *\|* Q\|+\epsilon$ for any real positive $\epsilon$. Thus $A \in \mathscr{Q}(S)$. In order to show that $\hat{A}$ extends $T$ we have to show that if $x \in H$ and $q \in V$ with $q \sim{ }^{*} x$, then $\Psi q \sim *^{*} x$. So assume $x=\Sigma_{k \in N} x_{k} e_{k} \in H$ and $q \in V$ such that ${ }^{*} x \sim q$. Then for every $\epsilon>0$ there exists $n$ such that $\left\|x-\Sigma_{k=1}^{n} x_{k} e_{k}\right\|=\alpha<\epsilon$. Hence ${ }^{*}\left\|{ }^{*} x-\sum_{k=1}^{n} x_{k}{ }^{*} e_{k}\right\|=\alpha$ and since ${ }^{*} e_{k} \sim q_{k}$ for each $k$ and $q \sim{ }^{*} x$, we have $*\left\|q-\sum_{k=1}^{n} x_{k} q_{k}\right\|==_{1} \alpha$, so $*\left\|\Psi q-\Sigma_{k=1}^{n} x_{k} p_{k}\right\|={ }_{1} \alpha$. Then $*\left\|\Psi q-\sum_{k=1}^{n} x_{k} e_{k}\right\|={ }_{1} \alpha$ and it follows that $*\left\|\Psi q-{ }^{*} x\right\|<2 \epsilon$ for every real positive $\epsilon$. Hence $\Psi q \sim{ }^{*} x$.

Now we show that $\hat{A}$ extends $T$. In order to do this it is sufficient to show that $\hat{A} e_{k}=T e_{k}$ for all $k \in N$. If $k \in N$, then $\hat{A} e_{k}=\hat{A} \pi\left(p_{k}\right)=\pi\left(A p_{k}\right)$. But $\Psi^{-1}\left(p_{k}\right)=q_{k}$ and ${ }^{*} Q\left(q_{k}\right) \sim{ }^{*} Q\left({ }^{*} e_{k}\right)={ }^{*}\left(T e_{k}\right)$, so $P_{V}^{*} Q q_{k} \sim{ }^{*}\left(T e_{k}\right)$. By the observation above $A p_{k}=\Psi P_{V}^{*} Q q_{k} \sim{ }^{*}\left(T e_{k}\right)$ and $\hat{A}\left(e_{k}\right)=T e_{k}$.

It remains to show that $\hat{A}$ is normal. Define $D$ on $S$ by $D p=$ $\Psi P_{V}^{*} Q^{\#} \Psi^{-1} p$. We assert that $D \sim A^{\#}$. To see this let $p, p^{\prime} \in S$ such that $*\|p\|=*\left\|p^{\prime}\right\|=1$. Then 


$$
\begin{aligned}
*\left\langle A p, p^{\prime}\right\rangle & ={ }^{*}\left\langle\Psi P_{V}{ }^{*} Q \Psi^{-1} p, p^{\prime}\right\rangle={ }_{1}{ }^{*}\left\langle P_{V}{ }^{*} Q \Psi^{-1} p, \Psi^{-1} p^{\prime}\right\rangle \\
& ={ }^{*}\left\langle{ }^{*} Q \Psi^{-1} p, \Psi^{-1} p^{\prime}\right\rangle={ }^{*}\left\langle\Psi^{-1} p,{ }^{*} Q^{\#} \Psi^{-1} p^{\prime}\right\rangle \\
& ={ }^{*}\left\langle\Psi^{-1} p, P_{V}{ }^{*} Q^{\#} \Psi^{-1} p^{\prime}\right\rangle={ }_{1}{ }^{*}\left\langle p, \Psi P_{V}{ }^{*} Q^{\#} \Psi^{-1} p^{\prime}\right\rangle={ }^{*}\left\langle p, D p^{\prime}\right\rangle .
\end{aligned}
$$

Hence $D \sim A^{\#}$.

Finally we show that $\hat{A}$ is normal. Since $\hat{A}^{\#}=\widehat{A^{\#}}=\hat{D}$, it is sufficient to show that for $p \in \operatorname{fin}\left({ }^{*} H\right) \cap S$ we have $A D p \sim D A p$. If $p \in \operatorname{fin}\left({ }^{*} H\right) \cap S$, then $A D p=\left(\Psi P_{V}^{*} Q \Psi^{-1}\right)\left(\Psi P_{V}{ }^{*} Q^{\#} \Psi^{-1} p\right)=\Psi P_{V}{ }^{*} Q P_{V}{ }^{*} Q^{\#} \Psi^{-1} p$. Since $P_{V}{ }^{*} Q \sim$ ${ }^{*} Q P_{V}$ we have $A D p \sim \Psi P_{V}{ }^{*} Q^{*} Q^{\#} \Psi^{-1} p$ and since $Q$ is normal $A D p \sim$ $\Psi P_{V}^{*} Q^{\# *} Q \Psi^{-1} p$. But $\Psi^{-1} p=P_{V} \Psi^{-1} p$ and $P_{V}^{*} Q \sim{ }^{*} Q P_{V}$, hence

$$
\begin{aligned}
A D p & \sim \Psi P_{V}^{*} Q^{\#} P_{V}^{*} Q \Psi^{-1} p \\
& =\Psi P_{V}^{*} Q^{\#} \Psi^{-1} \Psi P_{V}^{*} Q \Psi^{-1} p=D A p .
\end{aligned}
$$

This ends the proof of Theorem 2.10.

3. Spectrum of hyperfinite operators. We introduce the following notation. If $Q$ is an operator on a Hilbert space $H$, then $\Lambda(Q)$ is the spectrum of $Q, \Pi_{0}(Q)$ is the point spectrum of $Q$ (eigenvalues of $Q$ ), and $\Pi(Q)$ is the approximate point spectrum of $Q \quad \lambda \in \Pi(Q)$ if for every $\epsilon>0$ there exists $x \in H$ such that $\|x\|=$ 1 and $\|Q x-\lambda x\|<\epsilon)$. Note that if $\lambda \in \Lambda(Q) \backslash \Pi(Q)$ then the range of $Q-\lambda I$ is a proper closed subspace of $H$.

THEOREM 3.1. Let $S$ be $a{ }^{*}$-finite dimensional subspace of ${ }^{*} H$ such that $\hat{S} \supseteq H$ and let $A \in \mathscr{Q}(S)$. Then $\Lambda(\hat{A})=\Pi_{0}(\hat{A})$.

PRoof. First assume $\lambda \in \Pi(\hat{A})$. Then for every $n \in N$ there exists $p_{n} \in$ $S$ such that $*\left\|p_{n}\right\|=1$ and $*\left\|A p_{n}-\lambda p_{n}\right\|<1 / n$. It follows by $\aleph_{1}$-saturation that there exists $p \in S$ such that $*\|p\|=1$ and $A p \sim \lambda p$. Hence $\hat{A}(\pi(p))=$ $\lambda \pi(p)$ and $\lambda \in \Pi_{0}(\hat{A})$.

Now suppose there exists $\lambda \in \Lambda(A) \backslash \Pi(\hat{A})$. Then the range of $\hat{A}-\lambda I$ is a proper closed subspace of $\hat{S}$ so there exists $z \in \hat{S}$ such that $\|z\|=1$ and $\left\langle(\hat{A}-\lambda D x, z\rangle=0\right.$ for all $x \in \hat{S}$. Denote $A-\lambda I$ by $A_{\lambda}$. Pick $w \in S$ such that $*\|w\|=1$ and $\pi(w)=z$. Then $\left\langle A_{\lambda}(p), w\right\rangle={ }_{1} 0$ for all $p \in$ fin $\left({ }^{*} H\right) \cap S$. If $A_{\lambda}$ is not 1-1 on $S$, then since $S$ is *-finite and $A_{\lambda}$ is *-linear, there exists $p \in S$ such that $*\|p\|=1$ and $A_{\lambda} p=0$. But then $\hat{A}(\pi(p))=\lambda \pi(p)$ and $\lambda \in \Pi_{0}(\hat{A}) \subseteq$ $\Pi(A)$ which is a contradiction. Hence $A_{\lambda}$ is $1-1$ on $S$ and so for some $q \in S$ we have $A_{\lambda} q=w$. Now if $q \in$ fin $\left({ }^{*} H\right)$, then $1=*\|w\|^{2}=\left\langle A_{\lambda} q, w\right\rangle={ }_{1} 0$ which is impossible. But if $*\|q\|$ is infinite, then $A_{\lambda}[(1 / *\|q\|) q]=(1 / *\|q\|) w \sim 0$ and $(\hat{A}-\lambda I) \pi[(1 / *\|q\|) q]=0$. Again $\lambda \in \Pi_{0}(A)$ which is a contradiction. Thus $\Lambda(\hat{A})=\Pi(\hat{A})=\Pi_{0}(\hat{A})$. 
Note that if $\hat{A}$ is a hyperfinite extension of $T$, then $\Pi(T) \subseteq \Pi(\hat{A})=\Lambda(\hat{A})=$ $\Pi_{0}(\hat{A})$. For standard hyperfinite extensions we can say more.

THEOREM 3.2. If $\hat{T}_{S}$ is a standard hyperfinite extension of $T$, then $\Lambda(T)$ $\subseteq \Lambda\left(\hat{T}_{S}\right)=\Pi_{0}\left(\hat{T}_{S}\right)$.

Proof. By Theorem 3.1 we have $\Lambda\left(\hat{T}_{S}\right)=\Pi_{0}\left(T_{S}\right)$ and by the observation above $\Pi(T) \subseteq \Lambda\left(\hat{T}_{S}\right)$. Suppose $\lambda \in \Lambda(T) \backslash \Pi(T)$. Then there exists $z \in H$ such that $\|z\|=1$ and $\langle z,(T-\lambda I) x\rangle=0$ for all $x \in H$. Now if $p \in f i n\left({ }^{*} H\right) \cap S$, then $\left\langle{ }^{*} z,\left({ }^{*} T-\lambda I\right) p\right\rangle=0$ so $\left\langle P_{S}{ }^{*} z, P_{S}\left({ }^{*} T-\lambda I\right) p\right\rangle={ }_{1} 0$. Thus if $w=P_{S}{ }^{*} z$, then $\left\langle w,\left({ }^{*} T_{S}-\lambda I\right) p\right\rangle={ }_{1} 0$ for all $p \in$ fin $\left({ }^{*} H\right) \cap S$. Using the argument given above in the last part of the proof of Theorem 3.1, it follows that $\lambda \in \Pi_{0}(T)$.

EXAMPLES 3.3. (i) We give an example of a hyperfinite extension $\hat{A}$ of an operator $T$ such that $\Lambda(T) \nsubseteq \Lambda(A)$. Let $T$ be the shift operator on $l_{2}(N)\left(T e_{k}=\right.$ $e_{k+1}$ for all $\left.k \in N\right)$. Then $T$ is subnormal, indeed $T$ has an extension to the shift operator $Q$ on $l_{2}(Z)$. Since $Q$ is a unitary operator, it is easy to verify that in this case the hyperfinite extension $\hat{A}$ constructed in the proof of Theorem 2.10 is also a unitary operator. Thus $\Lambda(\hat{A}) \subseteq\{\lambda \in C:|\lambda|=1\}$, but $\Lambda(T)=\{\lambda \in C$ : $|\lambda| \leqslant 1\}$.

We give an example of an operator $T$ and a standard hyperfinite extension $\hat{T}_{S}$ such that $\Lambda(T) \neq \Lambda\left(\hat{T}_{S}\right)$. Let $T$ be the shift operator on $l_{2}(Z)$ and let $\hat{T}_{S}$ be the standard hyperfinite extension constructed in Example 2.9. Then $0 \in$ $\Lambda\left(\hat{T}_{S}\right) \backslash \Lambda(T)$.

THEOREM 3.4. If $T$ is quasitriangular then there exists a standard hyperfinite extension $\hat{T}_{S}$ such that $\Lambda(T)=\Lambda\left(\hat{T}_{S}\right)$.

Proof. Let $S$ be a *-finite dimensional subspace of ${ }^{*} H$ such that $\hat{S} \supseteq H$ and ${ }^{*} T P_{S} \sim P_{S}{ }^{*} T P_{S}$. If $\lambda \in \Lambda\left(\hat{T}_{S}\right)$ then by Theorem 3.1 there exists $p \in S$ such that ${ }^{*}\|p\|=1$ and $P_{S}{ }^{*} T p \sim \lambda p$. But ${ }^{*} T p \sim P_{S}{ }^{*} T p$, so ${ }^{*} T p \sim \lambda p$. It follows by a simple application of the transfer principle that $\lambda \in \Pi(T)$. Thus $\Lambda\left(\hat{T}_{S}\right) \subseteq$ $\Lambda(T)$ and by Theorem 3.2 we have $\Lambda\left(\hat{T}_{S}\right)=\Lambda(T)$.

Note that the proof of Theorem 3.4 shows that if $T$ is quasitriangular then $\Lambda(T)=\Pi(T)$. This was first shown by Deddens [3]. It is an interesting question whether for an arbitrary bounded operator $T$ there exists a standard hyperfinite extension $\hat{T}_{S}$ such that $\Lambda(T)=\Lambda\left(\hat{\Gamma}_{S}\right)$.

The following result is a partial answer to a question posed to the author by W. A. J. Luxemburg. If $S$ is a *-finite dimensional subspace of $* H$ and $A \in$ $\mathscr{U}(S)$, let $E(A)=\left\{\lambda \in{ }^{*} C\right.$ : there exists $p \in S$ with $*\|p\|=1$ and $\left.A p=\lambda p\right\}$, i.e. $E(A)$ is the set of *-eigenvalues of $A$.

THEOREM 3.5. If $T$ is quasitriangular there exists $a$ *-finite dimensional subspace $S$ with $\hat{S} \supseteq H$ and $A \in \mathscr{Y}(S)$ such that $A \sim{ }^{*} T_{S}$ and $\operatorname{st}(E(A))=\Lambda(T)$. 
PROOF. By Theorem 3.4 there exists a standard hyperfinite dimensional extension $\hat{T}_{S}$ of $T$ such that $\Pi_{0}\left(\hat{T}_{S}\right)=\Lambda\left(\hat{T}_{S}\right)=\Lambda(T)$. Assume $\Lambda(T)$ is infinite and pick a countable dense subset $\left\{\lambda_{k}: k \in N\right\}$ of $\Lambda(T)$.

We show first that for every $n \in N$ there exists $A_{n} \in \mathscr{M}(S)$ such that $A_{n} \sim$ ${ }^{*} T_{S}$ and $E\left(A_{N}\right) \supseteq\left\{\lambda_{1}, \lambda_{2}, \ldots, \lambda_{n}\right\}$. Since $\Pi_{0}\left(\hat{T}_{S}\right)=\Lambda\left(\hat{T}_{S}\right)=\Lambda(T)$, for each $k \in N$ there exists $x_{k} \in \hat{S}$ such that $\left\|x_{k}\right\|=1$ and $\hat{T}_{S}\left(x_{k}\right)=\lambda_{k} x_{k}$. For each $k$ pick $p_{k} \in S$ such that $*\left\|p_{k}\right\|=1$ and $\pi\left(p_{k}\right)=x_{k}$. Thus ${ }^{*} T_{S} p_{k} \sim \lambda_{k} p_{k}$ for each $k \in N$. Now let $n \in N$. Since $\left\{x_{1}, x_{2}, \ldots, x_{n}\right\}$ is linearly independent, it follows that $\left\{p_{1}, p_{2}, \ldots, p_{n}\right\}$ is *-linearly independent (Theorem 1.8 of [6]). Let $S_{n}=*_{\text {-span of }}\left\{p_{1}, p_{2}, \ldots, p_{n}\right\}$ and let $P_{n}$ be the *-projection of $S$ onto $S_{n}$.

Let $p \in$ fin $\left({ }^{*} H\right) \cap S_{n}$. Then $p=\Sigma_{k=1}^{n} a_{k} p_{k}$ and each $a_{k} \in$ fin $\left({ }^{*} R\right)$. If to the contrary some $a_{k}$ is infinite, let

$$
b=\max \left\{\left|a_{1}\right|,\left|a_{2}\right|, \ldots,\left|a_{n}\right|\right\} \text {. }
$$

Then $\Sigma_{k=1}^{n}\left(a_{k} / b\right) p_{k}=(1 / b) p \sim 0$ and so $\Sigma_{k=1}^{n}$ st $\left(a_{k} / b\right) x_{k}=0$ which contradicts the independence of the set $\left\{x_{1}, x_{2}, \ldots, x_{n}\right\}$, since for some $k,\left|\operatorname{st}\left(a_{k} / b\right)\right|$ $=1$. Define the *-linear mapping $Q_{n}$ on $S_{n}$ by $Q_{n}\left(\sum_{k=1}^{n} a_{k} p_{k}\right)=\sum_{k=1}^{n} a_{k} \lambda_{k} p_{k}$ for all $a_{1}, a_{2}, \ldots, a_{n} \in{ }^{*} C$ and set $A_{n}=Q_{n} P_{n}+{ }^{*} T_{S}\left(I-P_{n}\right)$. Now if $p \in S$ and $*\|p\|=1$, then $A_{n} p-{ }^{*} T_{S} p_{n}=\left(Q_{n}-{ }^{*} T_{S}\right) P_{n} p$. But $*\left\|P_{n} p\right\| \leqslant 1$, so $P_{n} p$ $=\Sigma_{k=1}^{n} a_{k} p_{k}$ where $a_{k}$ is finite for each $k$. Thus

$$
\left(Q_{n}-{ }^{*} T_{S}\right) p=\sum_{k=1}^{n} a_{k}\left(\lambda_{k} p_{k}-{ }^{*} T_{S} p_{k}\right) \sim 0
$$

since $\lambda_{k} p_{k} \sim{ }^{*} T_{S} p_{k}$ for each $k$. It follows that $A_{n} \sim{ }^{*} T_{S}$ and clearly $\left\{\lambda_{1}, \lambda_{2}\right.$, $\left.\ldots, \lambda_{n}\right\} \in E\left(A_{n}\right)$.

Hence for each $n \in N$ there exists $A_{n} \in \mathscr{Y}(S)$ such that $A_{n}\left(p_{k}\right)=\lambda_{k} p_{k}$ for $k=1,2, \ldots, n$ and $*\left\|* T_{S}-A_{n}\right\|<n^{-1}$. It follows by $\aleph_{1}$-saturation that there exists $A \in \mathscr{U}(S)$ such that $A \sim{ }^{*} T_{S}$ and $A p_{k}=\lambda_{k} p_{k}$ for all $k \in N$. Thus $E(A) \supseteq\left\{\lambda_{k}: k \in N\right\}$ and so $\operatorname{st}(E(A)) \supseteq\left\{\lambda_{k}: k \in N\right\}$. But since $E(A)$ is an internal set, $\operatorname{st}(E(A))$ is closed. Thus $\operatorname{st}(E(A)) \supseteq\left\{\overline{\lambda_{k}: k \in N}\right\}=\Lambda(T)$. On the other hand let $\lambda \in E(A)$. Since $|\lambda| \leqslant *\|A\| \leqslant *\left\|* T_{S}\right\|+1$, we have that $\lambda$ is finite. Pick $p \in S$ such that $*\|p\|=1$ and $A p=\lambda p$. Then $\hat{A} \pi(p)=\hat{T}_{S} \pi(p)=$ $\operatorname{st}(\lambda) \pi(p)$ and $\operatorname{st}(\lambda) \in \Pi_{0}\left(\hat{T}_{S}\right)$. Since $\Pi_{0}\left(\hat{T}_{S}\right)=\Lambda(T)$, we have that $\operatorname{st}(\lambda)$ is in $\Lambda(T)$. Hence $\operatorname{st}(E(A)) \subseteq \Lambda(T)$ and thus $\operatorname{st}(E(A))=\Lambda(T)$.

Finally note that if $\Lambda(T)$ is finite, say $\Lambda(T)=\left\{\lambda_{1}, \lambda_{2}, \ldots, \lambda_{n}\right\}$, then we may take $A=A_{n}$ above.

4. Spectral theorem for a bounded self adjoint operator. There have been previous nonstandard proofs of the spectral theorem for a bounded selfadjoint operator. In particular, there is a proof by A. Bernstein [1]. The interest of 
the proof presented here is that it is short and makes essential use of the external projection of $\hat{S}$ onto $H$.

Let $T$ be a bounded selfadjoint operator on $H$ and let $S$ be any *-finite dimensional subspace of ${ }^{*} H$ such that $\hat{S} \supseteq H$. Then ${ }^{*} T_{S}$ is ${ }^{*}$-selfadjoint on $S$ and $\hat{T}_{S}$ is a selfadjoint extension of $T$ to $\hat{S}$. Now if $V$ is a finite dimensional inner product space and $Q$ is a selfadjoint linear transformation of $V$ into itself, there exist an orthonormal basis $\left\{e_{1}, e_{2}, \ldots, e_{n}\right\}$ of $V$ and $\left\{\lambda_{1}, \lambda_{2}, \ldots, \lambda_{n}\right\} \subseteq R$ such that

(i) $\lambda_{1} \leqslant \lambda_{2} \leqslant \cdots \leqslant \lambda_{n}$ and

(ii) $Q\left(\sum_{i=1}^{n} a_{i} e_{i}\right)=\sum_{i=1}^{n} a_{i} \lambda_{i} e_{i}$ for all choices of $a_{1}, a_{2}, \ldots, a_{n}$ in $C$.

Hence there exists a ${ }^{*}$-orthonormal basis $\left\{\psi_{1}, \psi_{2}, \ldots, \psi_{\omega}\right\}$ for $S$ and a $*_{\text {-finite }}$ set $\left\{\lambda_{1}, \lambda_{2}, \ldots, \lambda_{\omega}\right\} \subseteq{ }^{*} R$ such that

(i) $\lambda_{i} \leqslant \lambda_{i+1}$ for $i=1,2, \ldots, \omega-1$ and

(ii) $* T_{S}\left(\sum_{i=1}^{\omega} a_{i} \psi_{i}\right)=\sum_{i=1}^{\omega} a_{i} \lambda_{i} \psi_{i}$ for any internal *-finite sequence $\left\{a_{1}\right.$, $\left.a_{2}, \ldots, a_{\omega}\right\} \subseteq{ }^{*} C$.

In particular ${ }^{*} T_{S} \psi_{i}=\lambda_{i} \psi_{i}$ and since ${ }^{*}\left\|T_{S}\right\| \leqslant\|T\|$ it follows that $\lambda_{i}$ is finite for $i=1,2, \ldots, \omega$. For each real $\mu$ and $n \in N$ define $S(\mu, n)$ to be the

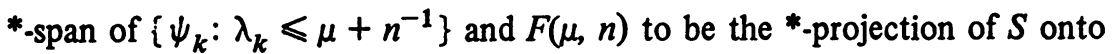
$S(\mu, n)$. Now $F(\mu, n)=0$ if $\lambda_{k}>\mu+n^{-1}$ for all $k$, and for each $\mu$ the sequence $\{\hat{F}(\mu, n)\}$ is a monotone decreasing sequence of projections on $\hat{S}$. Define $E(\mu)$ to be the strong limit of $\hat{F}(\mu, n)$. Then $\{E(\mu): \mu \in R\}$ is the spectral resolution for $\hat{T}_{S}$. More precisely:

THEOREM 4.1. (i) $E(\mu)=0$ if $\mu<\|\hat{T}\|$ and $E(\mu)=I$ if $\|\hat{T}\|<\mu$.

(ii) $E(\mu) E(\alpha)=E(\alpha) E(\mu)=E(\min (\alpha, \mu))$ for all $\mu, \alpha \in R$.

(iii) $E(\mu) \hat{T}_{S}=\hat{T}_{S} E(\mu)$ all $\mu \in R$,

(iv) $\alpha(E(\beta)-E(\alpha)) \leqslant \hat{T}_{S}(E(\beta)-E(\alpha)) \leqslant \beta(E(\beta)-E(\alpha))$ if $\alpha, \beta \in R$ with $\alpha<\beta$.

(v) $\lim _{\mu \rightarrow \alpha^{+}} E(\mu)=E(\alpha)$.

Proof. (i) This is immediate since st $\left(*\left\|* T_{S}\right\|\right)=\left\|\hat{T}_{S}\right\|=\|T\|$.

(ii) Let $\mu, \alpha \in R$ and $n \in N$, then

$$
F(\mu, n) F(\alpha, n)=F(\alpha, n) F(\mu, n)=F(\min (\mu, \alpha), n) .
$$

Hence $\hat{F}(\mu, n) \hat{F}(\alpha, n)=\hat{F}(\alpha, n) \hat{F}(\mu, n)=\hat{F}(\min (\mu, \alpha), n)$. Now taking limits we obtain (ii).

(iii) Let $\mu \in R$ and $n \in N$. Then $F(\mu, n){ }^{*} T_{S}={ }^{*} T_{S} F(\mu, n)$, hence $\hat{F}(\mu, n) \hat{T}_{S}=\hat{T}_{S} \hat{F}(\mu, n)$ and taking limits we obtain (iii).

(iv) Let $\alpha, \beta \in R$ with $\alpha<\beta$ and $n \in N$. Then

$$
\begin{aligned}
\left(\alpha+n^{-1}\right)(F(\beta, n)-F(\alpha, n)) & \leqslant{ }^{*} T_{S}(F(\beta, n)-F(\alpha, n)) \\
& \leqslant\left(\beta+n^{-1}\right)(F(\beta, n)-F(\alpha, n)) .
\end{aligned}
$$


Again (iv) follows by applying the ^ operation and taking limits.

(v) Finally if $\alpha \in R$ then $\{\hat{F}(\mu, n): \mu \in R, \mu>\alpha$ and $n \in N\}$ is cofinal with $\{\hat{F}(\alpha, n): n \in N\}$. Hence (v) holds.

Now let $P$ be the (external) projection of $\hat{S}$ onto $H$. Since $\hat{T}_{S}$ leaves $H$ invariant, it is easy to show that $P$ commutes with each projection $E(\mu)$. Hence if $G(\mu)$ is the restriction of $P E(\mu)$ to $H$ for each $\mu \in R$, then $\{G(\mu): \mu \in R\}$ is the spectral resolution for $T$.

\section{REFERENCES}

1. A. R. Bernstein, The spectral theorem-a nonstandard approach, Z. Math. Logik Grundlagen Math. 18 (1972), 419-434. MR 47 \#4048.

2. D. Cozart and L. C. Moore, Jr., The nonstandard hull of a normed Riesz space, Duke Math. J. 41 (1974), 263-275.

3. J. A. Deddens, A necessary condition for quasitriangularity, Proc. Amer. Math. Soc. 32 (1972), 630-631. MR 44 \#5810.

4. P. R. Halmos, Quasitriangular operators, Acta Sci. Math. (Szeged) 29 (1968), 283293. MR 38 \#2627.

5. Ten problems in Hilbert space, Bull. Amer. Math. Soc. 76 (1970), 877933. MR 42 \#5066.

6. C. Ward Henson and L. C. Moore, Jr., The nonstandard theory of topological vector spaces, Trans. Amer. Math. Soc. 172 (1972), 405-435; Erratum, ibid 184 (1973), 509. MR 46 \#7836; 48 \# 2708.

7. Subspaces of the nonstandard hull of a normed space, Trans. Amer. Math. Soc. 197 (1974), 131-143.

8. - Nonstandard hulls of the classical Banach spaces, Duke Math. J. 41 (1974), 277-284.

9. W. A. J. Luxemburg, A general theory of monads, Applications of Model Theory to Algebra, Analysis, and Probability (Internat. Sympos., Pasadena, Calif., 1967), Holt, Rinehart and Winston, New York, 1969, pp. 18-86. MR 39 \#6244.

10. A. Robinson, Non-standard analysis, North-Holland, Amsterdam, 1966. MR 34 \#5680.

DEPARTMENT OF MATHEMATICS, DUKE UNIVERSITY, DURHAM, NORTH CAROLINA 27706 